University of Nebraska - Lincoln

DigitalCommons@University of Nebraska - Lincoln

Faculty Publications from Nebraska Center for

Materials and Nanoscience, Nebraska Center Materials and Nanoscience

for (NCMN)

May 2007

\title{
Anisotropic strain and phonon deformation potentials in GaN
}

V. Darakchieva

IFM, Linköping University, SE-581 83 Linköping, Sweden

T. Paskova

Institute of Solid State Physics, University of Bremen, 28359 Bremen, Germany

Mathias Schubert

University of Nebraska - Lincoln, mschubert4@unl.edu

H. Arwin

IFM, Linköping University, SE-581 83 Linköping, Sweden

P.P. Paskov

IFM, Linköping University, SE-581 83 Linköping, Sweden

See next page for additional authors

Follow this and additional works at: https://digitalcommons.unl.edu/cmrafacpub

Part of the Nanoscience and Nanotechnology Commons

Darakchieva, V.; Paskova, T.; Schubert, Mathias; Arwin, H.; Paskov, P.P.; Monemar, B.; Hommel, D.; Heuken, M.; Off, J.; Scholz, F.; Haskell, B.A.; Fini, P.T.; Speck, J.S.; and Nakamura, S., "Anisotropic strain and phonon deformation potentials in GaN" (2007). Faculty Publications from Nebraska Center for Materials and Nanoscience. 36.

https://digitalcommons.unl.edu/cmrafacpub/36

This Article is brought to you for free and open access by the Materials and Nanoscience, Nebraska Center for (NCMN) at DigitalCommons@University of Nebraska - Lincoln. It has been accepted for inclusion in Faculty Publications from Nebraska Center for Materials and Nanoscience by an authorized administrator of DigitalCommons@University of Nebraska - Lincoln. 


\section{Authors}

V. Darakchieva, T. Paskova, Mathias Schubert, H. Arwin, P.P. Paskov, B. Monemar, D. Hommel, M. Heuken, J. Off, F. Scholz, B.A. Haskell, P.T. Fini, J.S. Speck, and S. Nakamura 


\title{
Anisotropic strain and phonon deformation potentials in GaN
}

\author{
V. Darakchieva,,${ }^{1, *}$ T. Paskova, ${ }^{2}$ M. Schubert,${ }^{3}$ H. Arwin,,${ }^{1}$ P. P. Paskov, ${ }^{1}$ B. Monemar,,${ }^{1}$ D. Hommel, ${ }^{2}$ M. Heuken, ${ }^{4}$ J. Off,,${ }^{5,}$ \\ F. Scholz,${ }^{5}$ B. A. Haskell,${ }^{6}$ P. T. Fini, ${ }^{6}$ J. S. Speck, ${ }^{6}$ and S. Nakamura ${ }^{6}$ \\ ${ }^{1}$ IFM, Linköping University, SE-581 83 Linköping, Sweden \\ ${ }^{2}$ Institute of Solid State Physics, University of Bremen, 28359 Bremen, Germany \\ ${ }^{3}$ Department of Electrical Engeneering, University of Nebraska, Lincoln, Nebraska 68588, USA \\ ${ }^{4}$ Aixtron AG, D-52072 Aachen, Germany \\ ${ }^{5} 4$ th Physical Institute, University of Stuttgart, 70569 Stuttgart, Germany \\ ${ }^{6}$ Materials Department, University of California, Santa Barbara, California 93106, USA
}

(Received 27 December 2006; revised manuscript received 21 March 2007; published 29 May 2007)

\begin{abstract}
We report optical phonon frequency studies in anisotropically strained $c$-plane- and $a$-plane-oriented GaN films by generalized infrared spectroscopic ellipsometry and Raman scattering spectroscopy. The anisotropic strain in the films is obtained from high-resolution x-ray diffraction measurements. Experimental evidence for splitting of the $\mathrm{GaN} E_{1}(\mathrm{TO}), E_{1}(\mathrm{LO})$, and $E_{2}$ phonons under anisotropic strain in the basal plane is presented, and their phonon deformation potentials $c_{E_{1}(T O)}, c_{E_{1}(L O)}$, and $c_{E_{2}}$ are determined. A distinct correlation between anisotropic strain and the $A_{1}(\mathrm{TO})$ and $E_{1}(\mathrm{LO})$ frequencies of $a$-plane GaN films reveals the $a_{A_{1}(T O)}, b_{A_{1}(T O)}$, $a_{E_{1}(L O)}$, and $b_{E_{1}(L O)}$ phonon deformation potentials. The $a_{A_{1}(T O)}$ and $b_{A_{1}(T O)}$ are found to be in very good agreement with previous results from Raman experiments [V. Yu. Davydov et al., J. Appl. Phys. 82, 5097 (1997)]. Our $a_{A_{1}(T O)}$ and $a_{E_{1}(L O)}$ phonon deformation potentials agree well with recently reported theoretical estimations [J.-M. Wagner and F. Bechstedt, Phys. Rev. B 66, 115202 (2002)], while $b_{A_{1}(T O)}$ and $b_{E_{1}(L O)}$ are found to be significantly larger than the theoretical values. A discussion of the observed differences is presented.
\end{abstract}

DOI: 10.1103/PhysRevB.75.195217

PACS number(s): 61.10.Nz, 63.20.-e, 81.05.Ea, 78.30.Fs

\section{INTRODUCTION}

Strain in semiconductors has a significant impact on the fundamental properties and characteristics of materials as it may change the crystal symmetry and changes the electronic band structure and phonon spectrum. Particularly appealing from the physical point of view are the anisotropically strained semiconducting layers, since they provide a possibility to study new effects, as well as impose some challenges on measurements and analyses. For instance, one of the most prominent effects of anisotropic strain on material properties is the change of the optical polarization selection rules as well as of the density of states in the valence band. Furthermore, optical phonons in uniaxial polar crystals exhibit a complex behavior under anisotropic strain. The phonons polarized in the plane perpendicular to the crystal optical axis are expected to shift and split under anisotropic strain, whereas the frequencies of the phonons polarized along the optical axis should only shift. The effects of strain on the frequencies and polarization directions of optical phonons can be employed as a tool for strain characterization experiments in crystalline semiconductor heterostructures. The method involves IR and Raman measurements of phonon frequency shifts with respect to unstrained material and their conversion into strain or stress components via the phonon deformation potentials. Knowledge of precise values of the phonon deformation potentials is of key importance in such studies. Phonon deformation potentials can be obtained from control experiments, in which the frequencies of the phonons under consideration are calibrated versus welldefined applied strain (or stress) fields on otherwise strainfree material. Alternatively, the phonon frequencies can be calibrated versus strain components in layers exhibiting different strain. The latter requires separate measurements of strain components.

Strain effects are particularly important for group-III nitrides because of the lack of a native substrate. The growth on foreign substrates typically leads to the presence of built-in strain in heteroepitaxial nitride layers due to the difference in lattice parameters and thermal expansion coefficients between layers and substrates. Sapphire and $\mathrm{SiC}$ are among the most often used substrates, and typically growth is realized on the basal (0001) c plane of sapphire and SiC. In such instances nitride films grow along the [0001] direction. The six fold symmetry of the basal planes of the hexagonal (nitrides, $\mathrm{SiC}$ ) and rhombohedral (sapphire) crystal structures dictates their isotropy in the basal plane and hence the thermal expansion coefficients and piezoelectric and elastic properties should be the same for any direction within the $c$ plane. As a consequence the films grown on $c$-plane substrates experience isotropic biaxial in-plane strain. Whenever growth is realized on non- $c$-plane-oriented sapphire and $\mathrm{SiC}$ surfaces the nitride films will be under anisotropic biaxial strain (independently of the film orientation) as a consequence of the anisotropy of the growth surfaces.

Recently the growth of group-III nitrides on non- $c$-plane substrates-e.g., nitride materials with nonpolar and semipolar surfaces ${ }^{1-5}$ - and $c$-plane $\mathrm{GaN}$ on $a$-plane sapphire ${ }^{6,7}$ has become of significant technological importance. The effect of the strain anisotropy on the optical response and electronic band structure has been experimentally studied for $c$-plane GaN films grown on $a$-plane sapphire, ${ }^{8,9} a$-plane GaN films grown on $r$-plane sapphire, ${ }^{10,11}$ and $m$-plane GaN films. ${ }^{12-14}$ The impact of anisotropic strain on the lattice parameters of 


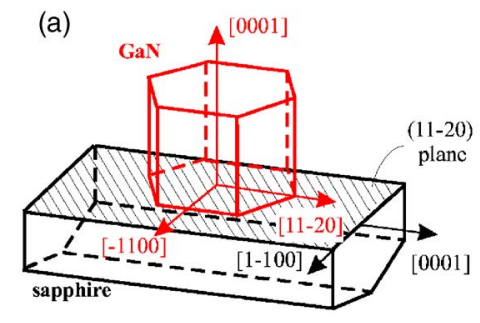

(c)

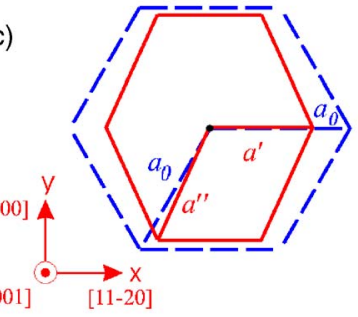

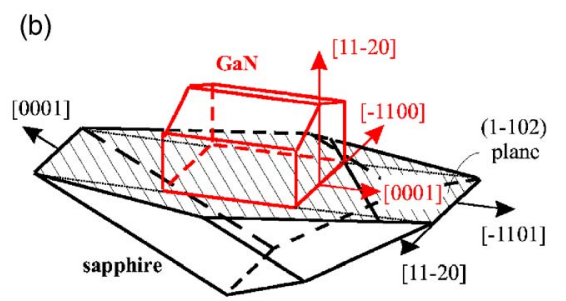

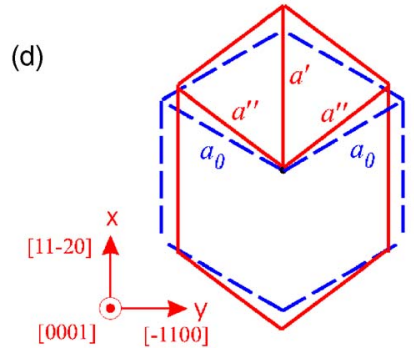

FIG. 1. (Color online) Schematic drawings of the relative arrangement of the unit cells and the orientations of the crystallographic axes of the (a) $c$-plane $\mathrm{GaN}$ on $a$-plane sapphire (hatched area) and (b) $a$-plane GaN on $r$-plane sapphire (hatched area). Distortions of the $\mathrm{GaN}$ basal plane under anisotropic strain: (c) $c$-plane $\mathrm{GaN}$ on $a$-plane sapphire and (d) $a$-plane $\mathrm{GaN}$ on $r$-plane sapphire. The undistorted basal planes are indicated by dashed lines in (c) and (d). $c$-plane GaN films grown on $a$-plane sapphire ${ }^{15}$ and on the structural properties of $a$-plane GaN (Refs. 16 and 17) has also been reported. In addition, anisotropic strain effects on the acoustic and surface properties of $m$-plane GaN films ${ }^{18,19}$ as well as detailed studies of the microstructure and structural properties of $a$ - and $m$-plane GaN (Refs. 1, 2, 20, and 21) and $c$-plane GaN films grown on $a$-plane sapphire ${ }^{22,23}$ were reported. In contrast, the vibrational properties of anisotropically strained $\mathrm{GaN}$ are poorly studied and no effect of anisotropic strain on phonons has been reported. There is no experimental evidence for splitting of $\mathrm{GaN}$ phonons under anisotropic strain, as theory predicts. Furthermore, there is no information on the phonon deformation potentials that quantify the effect of strain anisotropy in the basal hexagonal plane on the phonon frequency. In addition, the $E_{1}(\mathrm{LO})$ phonon deformation potentials have not been experimentally determined yet. Further, there is a lack of agreement between some phonon deformation potentials of GaN and AlN as determined by theory ${ }^{25}$ and by employing Raman scattering spectroscopy ${ }^{26,27}$ which makes further clarification of these issues by independent techniques highly desirable. This can be achieved by applying infrared spectroscopic ellipsometry (IRSE) to study the vibrational properties of non- $c$-axis oriented $\mathrm{GaN}$, since it allows access to the complete set of polar phonons in such samples.

In this work we report a comprehensive study of the vibrational properties of anisotropically strained $\mathrm{GaN}$ films. We employ generalized IRSE (GIRSE) and Raman scattering spectroscopy for the evaluation of phonon modes and highresolution x-ray diffraction (HRXRD) for determination of the anisotropic strain components. The effect of strain anisotropy on the GaN $E_{1}(\mathrm{TO}), E_{1}(\mathrm{LO})$, and $E_{2}$ phonons is found and discussed for $c$-plane GaN films grown on $a$-plane sapphire. The respective phonon deformation potentials, quantifying the effect of the anisotropy in the basal plane, are determined. The correlation between anisotropic strain and $A_{1}(\mathrm{TO})$ and $E_{1}(\mathrm{LO})$ phonon frequencies of $a$-plane $\mathrm{GaN}$ films was established and used to determine their deformation potentials by GIRSE. The results obtained are discussed in the context of previously reported theoretical and experimental [for the $A_{1}(\mathrm{TO})$ ] deformation potentials.

\section{EXPERIMENT}

We studied two types of anisotropically strained GaN layers: (i) $c$-plane GaN films grown on $a$-plane sapphire by metalorganic vapor phase epitaxy (MOVPE). The MOVPE films with thickness of about $2 \mu \mathrm{m}$ were grown in an Aixtron planetary reactor at $1170{ }^{\circ} \mathrm{C}$ using a low-temperature nucleation $\mathrm{GaN}$ layer. (ii) $a$-plane $\mathrm{GaN}$ films grown on $r$-plane sapphire by MOVPE and hydride vapor phase epitaxy (HVPE). In this case films with different thicknesses between $1 \mu \mathrm{m}$ and $67 \mu \mathrm{m}$ were studied. The $a$-plane MOVPE films were grown at $1030-1050{ }^{\circ} \mathrm{C}$ using a conventional two-step process employing GaN or AlN buffer layers. ${ }^{28}$ The $a$-plane HVPE films were grown directly on sapphire in a three-zone horizontal reactor at $1040-1070{ }^{\circ} \mathrm{C}^{29}$ Schematic drawings of the relative orientations of the anisotropically strained GaN films on their substrates are shown for $c$-plane $\mathrm{GaN}$ on $a$-plane sapphire in Fig. 1(a) and $a$-plane GaN on $r$-plane sapphire in Fig. 1(b), respectively.

HRXRD was performed using a Philips triple-axis diffractometer following the alignment procedure described in Ref. 30. Both $a$ and $c$ lattice parameters of the GaN layers were measured. The $c$ lattice parameters of all films were determined from the 0002, 0004, and $00062 \theta-\omega$ diffraction peaks. For the $c$-plane GaN films the measurements were performed at four different azimuthal positions achieved by a rotation of the sample about its normal by $90^{\circ}$. For the $a$-plane GaN films the measurements were performed in edge-symmetric geometry at two azimuths. The two $a$ lattice parameters in the case of $c$-plane GaN films were determined from the asymmetric $10 \overline{1} 4,10 \overline{1} 5,20 \overline{2} 4$, and $20 \overline{2} 5$ reflections at all six azimuth positions and the average value of the $c$ lattice parameter. In the case of $a$-plane $\mathrm{GaN}$ films the $a$ lattice parameter along the growth direction was determined from the $11 \overline{2} 0$ and $22 \overline{4} 0$ reflections for four azimuth positions separated by $90^{\circ}$. The second $a$ lattice parameter was determined from the $1 \overline{1} 00, \overline{1} 100,2 \overline{2} 00$, and $\overline{2} 200$ peaks measured in edge-symmetric geometry. For the $a$-plane $\mathrm{GaN}$ films the strain in the growth plane along the [1100] direction was estimated using the interplanar spacing, while for 
the estimation of the rest of the strains the values of the lattice parameters were used. The strain anisotropy in all films was estimated as the difference in the strains along the GaN $[11 \overline{2} 0]$ and [1 $\overline{1} 00]$ directions. In all estimations the lattice parameters reported in Ref. 31 were used as strain-free references. Further details about the lattice parameter determination of the $c$ - and $a$-plane $\mathrm{GaN}$ films can be found in previous work. ${ }^{15,17}$

The vibrational properties of all films were primarily studied by room-temperature GIRSE. The GIRSE measurements were performed on a rotating compensator infrared ellipsometer (J. A. Woollam Co, Inc.) in the spectral range of $350-1500 \mathrm{~cm}^{-1}$, with a spectral resolution of $2 \mathrm{~cm}^{-1}$, and at $60^{\circ}$ and $70^{\circ}$ angles of incidence. In the case of $c$-plane $\mathrm{GaN}$ films the measurements at each angle of incidence were performed for different angles $\varphi$ between the plane of incidence and the GaN [11 $\overline{2} 0]$ direction. For the $a$-plane GaN films the GIRSE spectra at each angle of incidence were taken at different angles $\varphi$ between the plane of incidence and the $c$ axes of the films.

Raman scattering measurements were also carried out in order to study the phonons of the $c$-plane $\mathrm{GaN}$ films grown on $a$-plane sapphire. The Raman spectra were taken at room temperature on a Dilor XY 800 spectrometer using the 514.5-nm line of an $\mathrm{Ar}^{+}$laser as excitation source. The spectral resolution was about $1 \mathrm{~cm}^{-1}$. The experiment was conducted in a backscattering configuration with the $z$ direction oriented along the film $c$ axis in several scattering geometries, allowing detection of the $E_{2}$ mode. ${ }^{26}$

\section{THEORY}

\section{A. Anisotropic strain}

The GaN films grown on $a$ - and $r$-plane sapphire are expected to experience anisotropic strain in the basal plane because of the difference in thermal expansion coefficients between sapphire and $\mathrm{GaN}$ and the different lattice mismatch along the two main in-plane directions [see Figs. 1(a) and 1(b)]. In linear elasticity theory the relation between the stress $\sigma$ and strain $\epsilon$ tensors is given by

$$
\begin{aligned}
\left(\begin{array}{c}
\sigma_{x x} \\
\sigma_{y y} \\
\sigma_{z z} \\
\sigma_{y z} \\
\sigma_{x z} \\
\sigma_{y z}
\end{array}\right)= & \left(\begin{array}{cccccc}
C_{11} & C_{12} & C_{13} & 0 & 0 & 0 \\
C_{12} & C_{11} & C_{13} & 0 & 0 & 0 \\
C_{13} & C_{13} & C_{33} & 0 & 0 & 0 \\
0 & 0 & 0 & C_{44} & 0 & 0 \\
0 & 0 & 0 & 0 & C_{44} & 0 \\
0 & 0 & 0 & 0 & 0 & 1 / 2\left(C_{11}-C_{12}\right)
\end{array}\right) \\
& \times\left(\begin{array}{c}
\epsilon_{x x} \\
\epsilon_{y y} \\
\epsilon_{z z} \\
2 \epsilon_{y z} \\
2 \epsilon_{x z} \\
2 \epsilon_{x y}
\end{array}\right)
\end{aligned}
$$

where $C_{i j}$ are the elastic stiffness constants and the $x, y$, and $z$ axes are chosen along the $\mathrm{GaN}[11 \overline{2} 0],[1 \overline{1} 00]$, and [0001] directions, respectively. Since these directions are parallel to the crystal principal axes, the shear stress and strain components $(i \neq j)$ are zero. ${ }^{32}$ Therefore, knowledge of the normal strain components $(i=j)$ is sufficient to fully describe the complex strain state in the $c$ - and $a$-plane $\mathrm{GaN}$ films that experience anisotropic basal distortions $\left(\epsilon_{x x} \neq \epsilon_{y y}\right)$.

The stress along the growth direction of the $c$-plane films, $\sigma_{z z}$, vanishes because the surface is free to expand or contract. It follows then from Eq. (1) that the strain along the growth direction can be expressed by the two in-plane strain components as

$$
\epsilon_{z z}=-\frac{C_{13}}{C_{33}}\left(\epsilon_{x x}+\epsilon_{y y}\right) .
$$

For the $a$-plane GaN films the growth is realized along the $[11 \overline{2} 0]$ direction and consequently $\sigma_{x x}$ vanishes. It follows from Eq. (1) that in this case

$$
\epsilon_{y y}=-\frac{C_{11}}{C_{12}} \epsilon_{x x}-\frac{C_{13}}{C_{12}} \epsilon_{z z} .
$$

\section{B. Optical phonon modes in GaN under anisotropic strain}

According to the factor group analysis, the optical phonon modes of wurtzite GaN belong to the following irreducible representation at the $\Gamma$ point of the Brillouin zone:

$$
\Gamma_{o p t}=\Gamma_{1}+2 \Gamma_{4}+2 \Gamma_{5}+\Gamma_{6}=A_{1}+2 B_{1}+E_{1}+2 E_{2} .
$$

The $A_{1}$ and $E_{1}$ modes are both IR and Raman active, the twofold $E_{2}\left(E_{2}^{\text {low }}\right.$ and $\left.E_{2}^{\text {high }}\right)$ are only Raman active, and the $B_{1}$ modes $\left(B_{1}^{\text {low }}\right.$ and $\left.B_{1}^{\text {high }}\right)$ are silent. Due to the macroscopic electric field associated with the relative atomic displacement of the longitudinal phonons, the polar $A_{1}$ and $E_{1}$ modes are split into LO and TO components.

The frequencies of the zone-center optical phonons in $\mathrm{GaN}$ are shifted or split with respect to the strain-free values. In the linear strain approximation the mode frequency changes are related to the strain tensor via ${ }^{24,25}$

$$
\begin{gathered}
\Delta \omega_{A_{1}}=a_{A_{1}}\left(\epsilon_{x x}+\epsilon_{y y}\right)+b_{A_{1}} \epsilon_{z z}, \\
\Delta \omega_{E_{1,2}}=a_{E_{1,2}}\left(\epsilon_{x x}+\epsilon_{y y}\right)+b_{E_{1,2}} \epsilon_{z z} \\
\pm c_{E_{1,2}}\left[\left(\epsilon_{x x}-\epsilon_{y y}\right)^{2}+4 \epsilon_{x y}^{2}\right]^{1 / 2} .
\end{gathered}
$$

The silent $B_{1}$ mode obeys a similar relation with strain as the $A_{1}$ mode. The coefficients $a_{j}, b_{j}$, and $c_{j}$ are the corresponding mode deformation potentials per unit strain.

\section{Generalized ellipsometry}

Ellipsometry determines the ratio of the complex reflection coefficients $R_{p}$ and $R_{s}$ for light polarized parallel $(p)$ and perpendicular $(s)$ to the plane of incidence, respectively, ${ }^{33}$ 


$$
\rho=R_{p} / R_{s}=\tan \Psi \exp (i \Delta),
$$

where $\Psi$ and $\Delta$ denote the ellipsometric angles. For our films it is necessary to apply a generalized ellipsometry approach since both $\mathrm{GaN}$ and sapphire are anisotropic materials and because of the non- $c$-axis orientation of the films and substrates. $^{30,34-36}$ In the generalized ellipsometry situation Eq. (7) depends on the polarization state of the incident plane wave and the respective parameters $\Psi_{i j}$ and $\Delta_{i j}(i, j=p, s)$ are defined by the following ratios of the polarized light reflection coefficients:

$$
\begin{aligned}
& \frac{r_{p p}}{r_{s s}}=R_{p p}=\tan \Psi_{p p} \exp \left(i \Delta_{p p}\right), \\
& \frac{r_{p s}}{r_{p p}}=R_{p s}=\tan \Psi_{p s} \exp \left(i \Delta_{p s}\right), \\
& \frac{r_{s p}}{r_{s s}}=R_{s p}=\tan \Psi_{s p} \exp \left(i \Delta_{s p}\right) .
\end{aligned}
$$

\section{Optical model}

A regression analysis is necessary in order to obtain the IR dielectric properties of the GaN films from the GIRSE data. The GIRSE data were modeled using a three-phase model: ambient/GaN layer/substrate for $\mathrm{GaN}$ films up to a thickness of $20 \mu \mathrm{m}$ and using a two-phase model and ambient/GaN layer for the thicker samples as the contribution from the substrate to the GIRSE response becomes negligible. A prerequisite for the data analysis is precise knowledge of the sapphire dielectric function, which was reported by Schubert et $a l .{ }^{37}$ and was taken without any changes in the present work. The anisotropic GaN dielectric functions were parametrized along Cartesian lattice directions: $j$ $=" x "=[11 \overline{2} 0], \quad j=" y "=[1 \overline{1} 00]$, and $j=" z "=[0001]$, accounting for the IR polar phonon mode contribution:

$$
\varepsilon_{j}^{L}(\omega)=\varepsilon_{\infty, j} \frac{\omega^{2}+i \gamma_{L O, j} \omega-\omega_{L O, j}^{2}}{\omega^{2}+i \gamma_{T O, j} \omega-\omega_{T O, j}^{2}}, \quad j=\text { “x, " “y," “ } z, "
$$

where $\omega_{T O, j=x, y, z}$ denote the frequencies of the $E_{1}^{x}(\mathrm{TO})$, $E_{1}^{y}(\mathrm{TO})$, and $A_{1}(\mathrm{TO})$, whereas $\omega_{L O, j=x, y, z}$ denote the frequencies of the $E_{1}^{x}(\mathrm{LO}), E_{1}^{y}(\mathrm{LO})$, and $A_{1}(\mathrm{LO})$ modes. $\gamma_{T O, j}$, and $\gamma_{L O, j}$ are the corresponding mode broadening parameters, and $\varepsilon_{\infty, x}, \varepsilon_{\infty, y}$, and $\varepsilon_{\infty, z}$ are the high-frequency limits for polarization perpendicular and parallel to the GaN $c$ axis. Note that in this presentation the $E_{1}$ modes may be different due to different strain the " $x$ " and " $y$ " directions-e.g., caused by anisotropic basal-plane distortion.

A possible contribution from free carriers to the dielectric functions of the $\mathrm{GaN}$ layers is also accounted for by virtue of the classical Drude model:

$$
\varepsilon_{j}^{(F C)}(\omega)=-\frac{\left(\omega_{p}^{*}\right)^{2}}{\omega\left(\omega+i \gamma_{p}\right)}, \quad j=“ x, " \text { “ } y, " \text { " } z, "
$$

with

$$
\omega_{p}^{*}=e \sqrt{\frac{N}{\varepsilon_{0} m}} .
$$

The isotropic unscreened plasma frequency $\omega_{p}^{*}$ depends on the free-charge-carrier concentration $N$ and the effective conduction band mass $m, \varepsilon_{0}$ is the vacuum permittivity, and $e$ is the electrical unit charge. The plasmon broadening parameter $\gamma_{p}$ is related to the optical free-charge-carrier mobility $\mu$ :

$$
\gamma_{p}=\frac{e}{m \mu} .
$$

Note that we treat both the effective mass and the mobility parameters isotropically.

In the case of $c$-plane-oriented films the model parameters that were allowed to vary during the GIRSE data analysis are the frequency and broadening parameters of the $E_{1}^{x}(\mathrm{TO})$, $E_{1}^{y}(\mathrm{TO}), E_{1}^{x}(\mathrm{LO}), E_{1}^{y}(\mathrm{LO})$, and $A_{1}(\mathrm{LO})$ modes, the highfrequency limit of the dielectric function, $\varepsilon_{\infty, j}$, the carrier concentration and mobility, and the thickness of the layers. Equal broadening parameters $\gamma_{T O, j}=\gamma_{L O, j}$ were assumed for $j=x, y$. For $c$-plane-oriented $\mathrm{GaN}$ the GIRSE data are not sensitive to the TO resonance frequency with polarization vector parallel to the sample normal. ${ }^{38}$ Consequently, the $A_{1}$ (TO) phonon mode resonance of the $c$-plane GaN could not be found and the values determined from Raman measurements were used in the modeling of the GIRSE data as fixed parameters. In the case of $a$-plane-oriented films the best-match model parameters are the frequency and broadening parameters of the $E_{1}^{x}(\mathrm{TO}), E_{1}^{y}(\mathrm{TO}), A_{1}(\mathrm{TO}), E_{1}^{x}(\mathrm{LO})$, $E_{1}^{y}(\mathrm{LO})$, and $A_{1}(\mathrm{LO})$ modes, the high-frequency limit of the dielectric function, $\varepsilon_{\infty, j}$, the carrier concentration and mobility, and the thickness of the layer. For some of the $a$-plane films the GIRSE data possess little sensitivity to the $E_{1}^{y}$ frequency or to the difference between the frequencies and broadening parameters of the phonons polarized along the $[11 \overline{2} 0]$ and $[1 \overline{100}]$ directions, respectively. Consequently, the $E_{1}^{i}$ phonon parameters and high-frequency limits of the dielectric constant were treated isotropically in these cases. Further details about the data analysis can be found in Ref. 33 and references therein.

\section{RESULTS AND DISCUSSION}

\section{A. Anisotropic strain}

We have experimentally determined two different $a$ lattice parameters for our GaN films as expected in the presence of anisotropic biaxial strain. In the case of $c$-plane-oriented films we found that the $2 \theta-\omega$ peak of the HRXRD asymmetric scans appears at different positions when measured at the six azimuthal positions which reflects an anisotropic distortion of the basal plane. ${ }^{15}$ Four of the $2 \theta-\omega$ positions appear at lower and two at higher scattering angles. The values of the interplanar distances derived from these peak positions can be discriminated accordingly into two groups, and consequently, the in-plane lattice parameter has two different values. ${ }^{15} \mathrm{We}$ found that the two $a$ lattice parameters are smaller than the strain-free value ${ }^{31}$ as one can expect from the difference of the thermal expansion coefficients between 
TABLE I. Values of the experimentally determined strain components in the $c$ - and $a$-plane GaN films

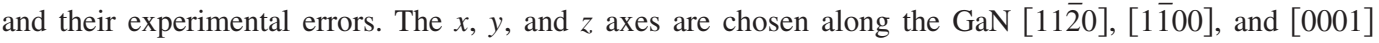
directions, respectively.

\begin{tabular}{lccc}
\hline \hline Sample & $\epsilon_{x x}$ & $\epsilon_{y y}$ & $\epsilon_{z z}$ \\
\hline $1.7 \mu \mathrm{m} c-\mathrm{GaN}$ & $-2.29 \pm 0.5 \times 10^{-3}$ & $-1.63 \pm 0.7 \times 10^{-3}$ & $8.23 \pm 0.4 \times 10^{-4}$ \\
$1.2 \mu \mathrm{m} a-\mathrm{GaN}$ & $4.32 \pm 0.8 \times 10^{-4}$ & $-4.01 \pm 1.8 \times 10^{-4}$ & $-9.05 \pm 0.6 \times 10^{-4}$ \\
$1.5 \mu \mathrm{m} a-\mathrm{GaN}$ & $0.76 \pm 0.8 \times 10^{-4}$ & $-8.42 \pm 1.8 \times 10^{-4}$ & $-1.03 \pm 0.14 \times 10^{-3}$ \\
$17 \mu \mathrm{m} a-\mathrm{GaN}$ & $9.60 \pm 0.4 \times 10^{-4}$ & $-1.43 \pm 0.18 \times 10^{-3}$ & $-9.39 \pm 0.5 \times 10^{-4}$ \\
$34 \mu \mathrm{m} a-\mathrm{GaN}$ & $8.88 \pm 0.6 \times 10^{-4}$ & $-1.28 \pm 0.18 \times 10^{-3}$ & $-7.02 \pm 1.1 \times 10^{-4}$ \\
$60 \mu \mathrm{m} a-\mathrm{GaN}$ & $6.92 \pm 0.5 \times 10^{-4}$ & $-1.02 \pm 0.18 \times 10^{-3}$ & $-0.26 \pm 2.5 \times 10^{-4}$ \\
\hline \hline
\end{tabular}

GaN and sapphire. ${ }^{39}$ The distortion of the GaN basal plane under such an anisotropic compressive strain is illustrated in Fig. 1(c). The $c$ lattice parameters of the $c$-GaN films were found to be larger than the strain-free value as the elasticity theory predicts in the case of biaxial strain. The estimated out-of-plane and in-plane strain components of one representative $c$-plane $\mathrm{GaN}$ film are given in Table I. It is worth mentioning that we have also determined two different $a$ lattice parameters for HVPE GaN films grown on $a$-plane sapphire, in agreement with the results for the MOVPE films. However, in this case the thickness of the films was between $40 \mu \mathrm{m}$ and $60 \mu \mathrm{m}$, which results in a smaller difference between the two $a$ lattice parameters-i.e., smaller strain anisotropy. Consequently, the effect of anisotropic strain on the GaN phonons was difficult to detect and therefore the HVPE films were not considered in the current study.

Similarly to the $c$-oriented films, we found that all $a$-plane GaN films are compressively strained in the growth plane (both along the [0001] and [1 100$]$ directions) and the strain along the growth direction is tensile [see Fig. 1(b)]. The respective strain components of the $a$-plane films are given in Table I. The strain in this case, however, results in a more complex distortion of the basal GaN plane, which is compressed along the $[1 \overline{1} 00]$ and expanded along the $[11 \overline{2} 0]$ directions as illustrated in Fig. 1(d).

A comparison between the experimentally determined and theoretical values of $\epsilon_{z z}$ estimated by Eq. (2) gives excellent agreement for the $c$-plane-oriented films. The experimentally determined and theoretical values of $\epsilon_{y y}$ estimated via Eq. (3) for the $a$-plane-oriented films agree fairly well. We note, however, that generally the edge geometry used in the experimental determination of $\epsilon_{y y}$ and $\epsilon_{z z}$ leads to larger uncertainties compared to the symmetric geometry used for the determination of $\epsilon_{x x}$. In addition uncertainties in the values of the in-plane strains are introduced due to the fact that different thicknesses are probed (in particular for the thicker films) in the normal and edge geometries. Consequently, the experimentally and theoretically estimated $\epsilon_{y y}$ values may deviate up to $20 \%$ for some of the samples. In all theoretical estimations the stiffness constants of GaN reported in Ref. 40 were used.

\section{B. Anisotropic phonon deformation potentials}

Figure 2 shows experimental and best-fit model calculated $\Psi_{i j}$ spectra for one representative $c$-plane GaN layer on $a$-plane sapphire. The GIRSE spectra of the $c$-plane GaN films are dominated by the reststrahlen bands of sapphire, indicated by the brackets below the spectra in Fig. 2. The sharp peaks in $\Psi_{i j}$ spectra within the sapphire reststrahlen range (300-980 $\mathrm{cm}^{-1}$ ) are due to bands of total reflection for the $p$ - and $s$-polarized light components between the sapphire $E_{u}$ and $A_{2 u}$ phonon modes. The lattice modes of the GaN films also affect the $\Psi_{i j}$ spectra. In particular, the GIRSE spectra provide high sensitivity to the frequency and broadening parameters of the $\mathrm{GaN} E_{1}^{j}(\mathrm{TO})$ modes. It is seen from Fig. 2 that a distinct spectral feature in $\Psi_{p p}$ appears near the GaN $E_{1}(\mathrm{TO})$ resonance, which changes in a subtle manner upon rotation of the sample by $\varphi$. Spectral features are related to the $\mathrm{LO}$ modes and $A_{1}(\mathrm{LO})$ and $E_{1}^{j}(\mathrm{LO})$ phonon mode parameters were obtained from the line-shape analysis. The free-carrier concentration in all $c$-plane GaN films studied was found to be below the detection limit of the GIRSE technique $\left.\left(<1 \times 10^{17} \mathrm{~cm}^{-3}\right)\right)^{38}$

Figure 3 depicts the best-match anisotropic model dielectric function spectra together with the imaginary parts of their respective dielectric loss functions obtained from a representative $c$-plane GaN sample grown on $a$-plane sapphire. The $z$ direction response is along the $\mathrm{GaN} c$ axis, whereas the $x$ and $y$ directions are parallel to the $\mathrm{GaN}[11 \overline{2} 0]$ and $[\overline{1} 100]$ directions, respectively. The different strains along the $x$ and $y$ axes result in different phonon mode shifts and cause spectrally narrow differences between the imaginary parts within the phonon mode resonance region (dichroism). Causality requirements then produce birefringence over a much larger spectral range than imposed by the phonon mode shifts, which also affects the imaginary parts of the dielectric loss functions. Despite the fact that the spectral resolution of the ellipsometer system employed in this work is larger than the transverse optical phonon mode frequency shifts, the phonon mode resonance shifts can be located precisely by our model line-shape analysis of the GIRSE data. A more detailed discussion of this effect is given in the Appendix.

The frequencies and the broadening parameters of the $E_{1}$ phonons polarized in the $x-y$ plane parallel to the $\mathrm{GaN}$ $[11 \overline{2} 0]$ and $[\overline{1} 100]$ directions, extracted from the best-match modeling to the experimental GIRSE data, are given in Table II. The phonon mode parameters for both polarizations were determined with high precision, much better than the spectral resolution. The use of an appropriate line shape to describe the IR response and its cause is mandatory to allow resolv- 

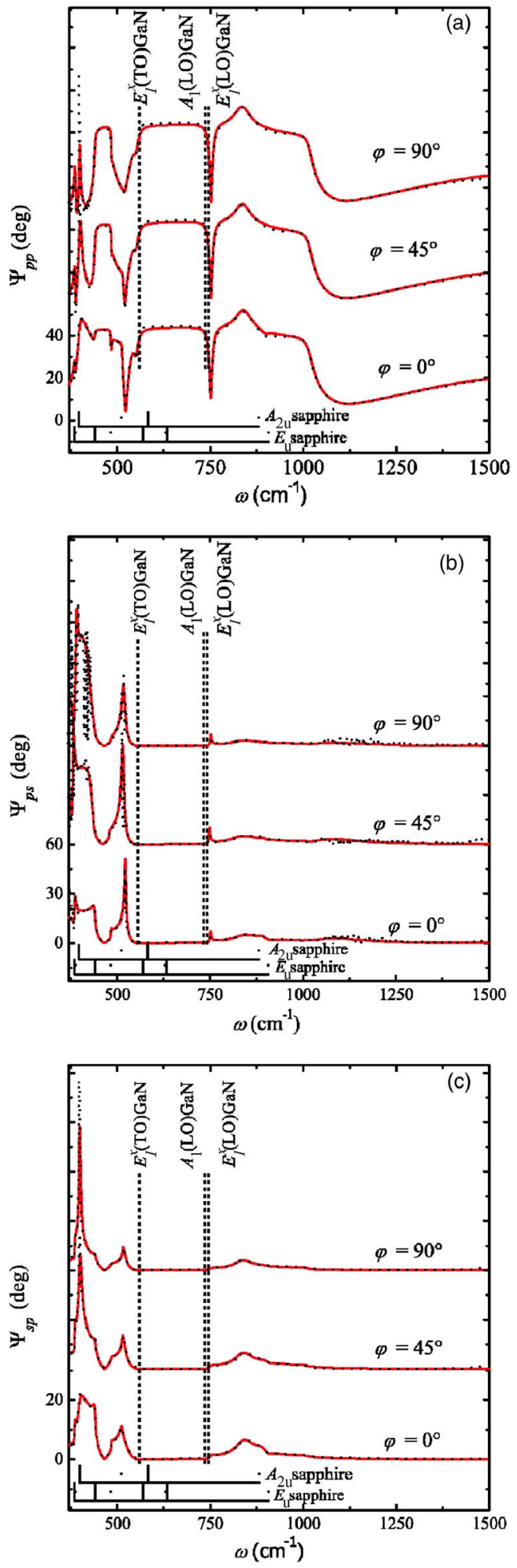

FIG. 2. (Color online) Experimental (dots) and calculated (lines) GIRSE spectra of one representative $c$-plane GaN film on $a$-plane sapphire for different angles $\varphi$ between the plane of incidence and the GaN [11 $\overline{2} 0$ ] direction: (a) $\Psi_{p p}$, (b) $\Psi_{p s}$, and (c) $\Psi_{s p}$. The strainfree frequencies of the $\mathrm{GaN}$ phonon modes of $A_{1}$ and $E_{1}$ symmetry are indicated by dashed lines. For the sake of clarity the phonon frequencies are given only for polarization along the [11 $\overline{2} 0]$ direction. The $A_{2 u}$ and $E_{u}$ phonon modes of sapphire are indicated in brackets.

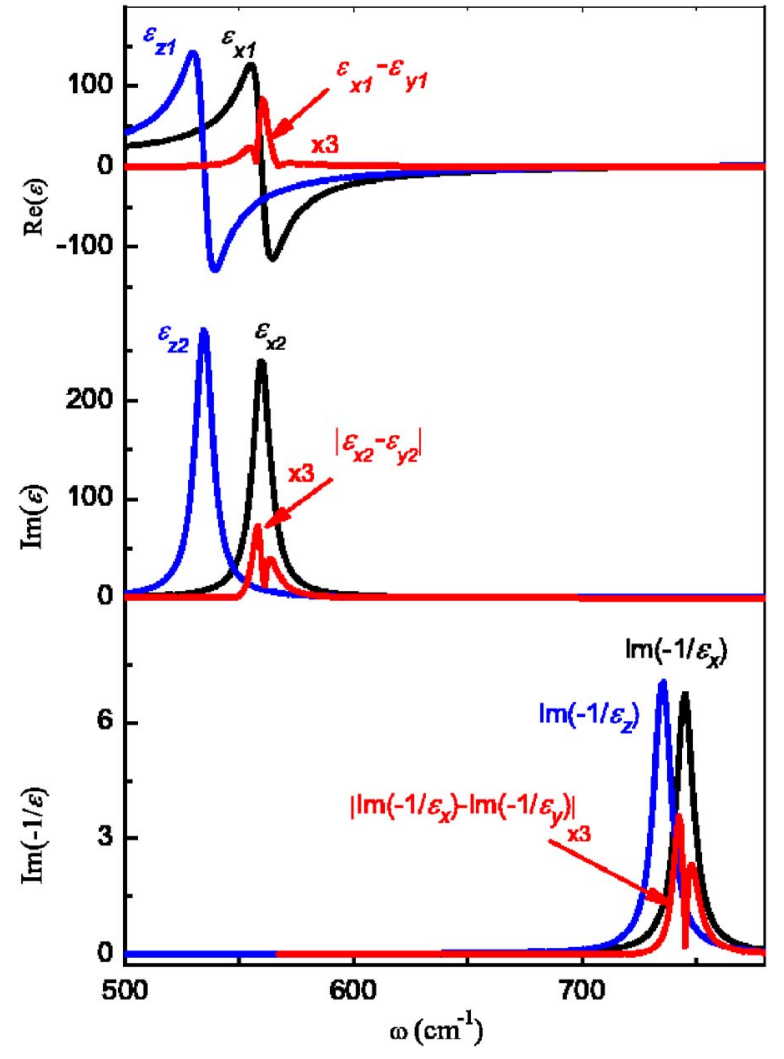

FIG. 3. (Color online) Real and imaginary parts of the dielectric function $\varepsilon$ and imaginary part of the dielectric loss functions $-1 / \varepsilon$ for in- $(x)$ and out-of-plane $(z)$ directions of one representative $c$-plane GaN film grown on $a$-plane sapphire surface. For clarity, the functions for directions $y$ are given through the differences between those for directions $(x)$ parallel to the $\mathrm{GaN}[11 \overline{2} 0]$ and $(y)$ parallel to the $\mathrm{GaN}[1 \overline{1} 00]$ directions.

ability of the phonon parameters when the resolution is larger than the error bars of the respective parameter. We have shown previously that the Lorentzian-broadened harmonic oscillator describes very accurately the polar phonon contribution to the IR response for GaN. ${ }^{38}$ The accurate location of the center energy of the Lorentzian-broadened harmonic oscillator is possible since its broadening is sufficiently larger than the spectral resolution.

As seen from Table II, a small difference in the frequency values of the $E_{1}$ phonons was determined for polarizations along the $\mathrm{GaN}[11 \overline{2} 0]$ and $[\overline{1} 100]$ directions upon the model layer calculations. This experimental finding agrees with the theoretical predictions that the frequencies of the $E_{1}$ phonons in GaN are split when $\epsilon_{x x} \neq \epsilon_{y y}$ [see Eq. (6)]. ${ }^{24,25}$ It can be seen from Table II that the broadening parameters of the $E_{1}$ phonons polarized along the $[11 \overline{2} 0]$ and [1 $\overline{100}]$ directions also show a difference of about $0.6 \mathrm{~cm}^{-1}$. The $E_{1}^{x}(\mathrm{TO})$ and $E_{1}^{x}(\mathrm{LO})$ exhibit larger broadening, which could be related to the larger compressive in-plane strain along the $\mathrm{GaN}$ [11 $\overline{2} 0]$ direction as compared to the $[1 \overline{1} 00]$ direction (Table I). We have also extracted from the best fit to the GIRSE data the high-frequency dielectric constant along the [11 $\overline{2} 0]$ and [1 100$]$ directions to be $5.18 \pm 0.01$ and $5.15 \pm 0.01$, respec- 
TABLE II. Best-fit values and standard deviations of the $E_{1}^{j}(\mathrm{TO}), E_{1}^{j}(\mathrm{LO})$, and $E_{2}^{j}$ phonon frequencies $\omega$ and broadening parameters $\gamma$ for polarizations along the $\operatorname{GaN}[11 \overline{2} 0](j=$ " $x ")$ and $[\overline{1} 100]$ ( $j=$ " $y ")$ directions and the respective phonon deformation potentials $c$. For $E_{1}$ modes $j$ denotes the phonon polarization, whereas for $E_{2}$ modes $j$ denotes the polarization of the incident light in the parallel and cross-polarized configurations, respectively.

\begin{tabular}{lccccc}
\hline \hline & \multicolumn{2}{c}{$\omega\left(\mathrm{cm}^{-1}\right)$} & \multicolumn{2}{c}{$\gamma\left(\mathrm{cm}^{-1}\right)$} \\
\cline { 2 - 4 } & $j=[11 \overline{2} 0]$ & $j=[1 \overline{1} 00]$ & $j=[11 \overline{2} 0]$ & $j=[1 \overline{1} 00]$ & \multirow{2}{c}{$c\left(\mathrm{~cm}^{-1}\right)$} \\
\hline$E_{1}^{j}(\mathrm{TO})$ & $559.8 \pm 0.04$ & $559.3 \pm 0.04$ & $9.2 \pm 0.1$ & $8.6 \pm 0.07$ & $379 \pm 43$ \\
$E_{1}^{j}(\mathrm{LO})$ & $745.2 \pm 0.05$ & $744.3 \pm 0.04$ & $9.2 \pm 0.1$ & $8.6 \pm 0.07$ & $678 \pm 49$ \\
$E_{2}^{j}$ & $570.4 \pm 0.1$ & $569.9 \pm 0.1$ & $1.6 \pm 0.03$ & $1.7 \pm 0.06$ & $379 \pm 107$ \\
\hline \hline
\end{tabular}

tively. This subtle anisotropy of the dielectric constant in the $x-y$ plane may likely be ascribed to strain-related effects also. The in-plane dielectric constants are both smaller than $\varepsilon_{\infty, z}$ along the [0001] direction, determined to be $5.28 \pm 0.01$, in agreement with previous theoretical and experimental findings for GaN. ${ }^{25,38}$

In order to study the behavior of the $\mathrm{GaN} E_{2}$ phonon we performed Raman scattering spectroscopy in parallel, $z(x x) \bar{z}$, and cross-polarized, $z(y x) \bar{z}$, scattering geometries, allowing measurements with incident polarization along the $\mathrm{GaN}$ $[11 \overline{2} 0]$ and $[1 \overline{100}]$ directions, respectively. Figure 4 shows Raman spectra from one representative $c$-plane GaN film in the vicinity of the GaN $E_{2}$ phonon mode. Experimental values of the $E_{2}$ frequency and broadening parameters were extracted from the Raman spectra using a standard bestmatch procedure with Lorentzian line shapes. The bestmatch results are shown in Fig. 4, and the extracted phonon parameters are listed in Table II. A subtle difference in the $E_{2}$ frequency can be determined when measuring in $z(x x) \bar{z}$ and $z(y x) \bar{z}$ configurations. Although the $E_{2}$ frequency positions for $[1 \overline{1} \overline{0}]$ and $[1 \overline{1} 00]$ polarizations differ by less than

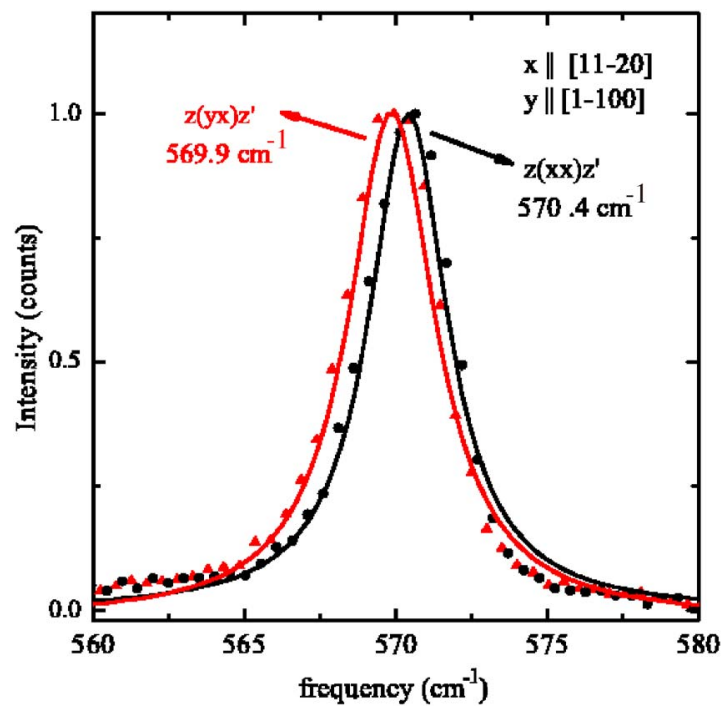

FIG. 4. (Color online) Raman spectra in parallel $(x x)$ and crosspolarized $(y x)$ scattering geometries from one representative $c$-plane GaN film on $a$-plane sapphire in the vicinity of the $\mathrm{GaN} E_{2}$ mode.
$1 \mathrm{~cm}^{-1}$, the observed splitting of the $E_{2}$ phonon was reproducible. It is concordant with the theoretical prediction ${ }^{24,25}$ for the $E_{2}$ behavior under anisotropic basal strain [Eq. (6)] and with our findings for the splitting of the polar phonons polarized along the two in-plane directions. We note that splitting of the $E_{2}$ mode in the Raman spectra of wurtzite polar crystals with $\epsilon_{x x} \neq \epsilon_{y y}-$ e.g., $\mathrm{CdS}$ - has been reported. ${ }^{24}$

The shear strain component $\epsilon_{x y}$ for our samples is zero, and it follows from Eq. (6) that the splittings of the $E_{1}$ and $E_{2}$ phonons depend only on the magnitude of the in-plane strain anisotropy, $\epsilon_{x x}-\epsilon_{y y}$. Consequently, the difference between the frequencies of the $E_{1,2}$ phonons for polarizations along the $\mathrm{GaN}[11 \overline{2} 0]$ and [ $\overline{1} 100]$ directions is given by

$$
\omega\left(E_{1,2}\right)_{[11 \overline{1} 0]}-\omega\left(E_{1,2}\right)_{[1 \overline{1} 00]}=2 c\left(E_{1,2}\right)\left|\epsilon_{x x}-\epsilon_{y y}\right| .
$$

Using our experimental results for the strain components and the phonon frequency splittings we have estimated the $c$ phonon deformation potentials for the $E_{1}(\mathrm{TO}), E_{1}(\mathrm{LO})$, and $E_{2}$ phonons. The results are listed in Table II. The difference between the $c$ deformation potentials of the $E_{1}(\mathrm{TO})$ $\left(379 \mathrm{~cm}^{-1}\right)$ and $E_{1}(\mathrm{LO})\left(682 \mathrm{~cm}^{-1}\right)$ modes is relatively large, which can be related to the large bond ionicity in GaN causing substantial mode polarity. In some rare instances the $E_{2}$ splitting was determined to be as low as $0.3 \mathrm{~cm}^{-1}$ and therefore the estimated $c_{E_{2}}\left(379 \mathrm{~cm}^{-1}\right)$ given in Table II should be regarded as an upper limit. We further found that an $a$-plane GaN film, exhibiting significant strain anisotropy in the basal plane, revealed $E_{1}(\mathrm{TO})$ and $E_{2}$ frequency splittings of $2 \mathrm{~cm}^{-1}$ in the respective Raman spectra (not shown here). A correlation of these splittings with the strain anisotropy in the basal plane of the films results in $c_{E_{2}}$ and $c_{E_{1}(T O)}$ of $369 \mathrm{~cm}^{-1}$, in excellent agreement with the results for the anisotropically strained $c$-plane films (Table II).

The lack in the existing literature of any theoretical and experimental values for the $c$ phonon deformation potentials of $\mathrm{GaN}$ and other III-nitrides renders comparative analysis of our results with existing data difficult. By simple argument, however, the high bond strength of $\mathrm{GaN}$ is expected to result in relatively large values of the $c$ phonon deformation potentials as compared to II-VI materials with the same crystal symmetry. A comparison with $\mathrm{CdS}$, for example, reveals that the GaN $c$ phonon deformation potentials are found to be significantly larger than in $\mathrm{CdS}$, concordant with the argumentation above. ${ }^{24}$ 


\section{C. $A_{1}(\mathrm{TO})$ and $E_{1}(\mathrm{LO})$ phonon deformation potentials}

Experimental and best-fit model-calculated $\Psi_{i j}$ spectra for one representative MOVPE $a$-plane GaN layer on $r$-plane sapphire are shown in Fig. 5. The coupling between $p$ - and $s$-polarized light, sensed by the off-diagonal elements, is small at $\varphi=0^{\circ}$ and minimal at $\varphi=90^{\circ}$ [Figs. 5(b) and 5(c)]. A noticeable conversion of $p-(s-)$ to $s^{-}(p-)$ polarized light related to the GaN film is observed in the GIRSE spectra for intermediate $\varphi$, particularly across frequency regions of resonant excitation of TO or LO phonons. It can be seen that the low-frequency edge of the GaN reststrahlen band in $\Psi_{p p}$ moves from $E_{1}(\mathrm{TO})$ to $A_{1}(\mathrm{TO})$ when the azimuth angle $\varphi$ between the plane of incidence and the $\mathrm{GaN} c$ axis changes between $0^{\circ}$ and $90^{\circ}$ [Fig. 5(a)]. The $E_{1}(\mathrm{TO})$ and $A_{1}(\mathrm{TO})$ are excited resonantly depending on $\varphi$ as the electric field parallel to the film interface senses lattice excitations with polarization perpendicular or parallel to the $\mathrm{GaN} c$ axis, respectively.

We extracted from the best fit to the GIRSE data the frequencies and broadening parameters of the polar phonons, as well as the free-carrier concentrations and mobilities for all samples. The free-carrier concentration in the $a$-plane films grown by MOVPE was found to be below the detection limit of the GIRSE technique $\left(<1 \times 10^{17} \mathrm{~cm}^{-3}\right){ }^{38}$ On the other hand, the films grown by HVPE show a free-carrier concentration of the order of $10^{18} \mathrm{~cm}^{-3}$, exhibiting complex behavior across the film thickness. ${ }^{41}$ This resulted in larger uncertainties in the LO phonon frequencies compared to the TO phonons.

We focus our attention on the $A_{1}$ (TO) phonons, since there is so far only one experimental work on the $A_{1}(\mathrm{TO})$ deformation potentials, based on Raman scattering, ${ }^{26}$ that contradicts recent theoretical estimations. ${ }^{25}$ The $A_{1}$ (TO) frequency extracted from the GIRSE analysis changes in a complex manner with film thickness following the change in $\epsilon_{x x}$ and $\epsilon_{z z}$ (Fig. 6). To obtain the deformation potentials and strain-free frequency from our results it is necessary to perform a regression analysis of the experimental frequencies and strains. However, Eq. (5) cannot be directly used as the three strain components are not independent. Substituting Eq. (3) into Eq. (5) we obtain

$$
\Delta \omega_{A_{1}(T O)}=a_{A_{1}(T O)}\left(1-\frac{C_{11}}{C_{12}}\right) \epsilon_{x x}-\left(a_{A_{1}(T O)} \frac{C_{13}}{C_{12}}-b_{A_{1}(T O)}\right) \epsilon_{z z} .
$$

Then using our experimental results about the $A_{1}(\mathrm{TO})$ frequency and the strain components $\epsilon_{x x}$ and $\epsilon_{z z}$ we performed a regression analysis of Eq. (16). The values obtained of the phonon deformation potentials and the strain-free frequency of the $\mathrm{GaN} A_{1}(\mathrm{TO})$ mode and their standard deviations are listed in Table III together with previous theoretical and experimental results. The contour plot in Fig. 6 demonstrates the dependence of the $A_{1}(\mathrm{TO})$ frequency on the in-plane strain components $\epsilon_{x x}$ and $\epsilon_{z z}$ according to Eq. (16) and using our results about the phonon deformation potentials and strain-free frequency. The squares show the calculated frequencies using our measured $\epsilon_{x x}$ and $\epsilon_{z z}$, and the respective labels give the frequency values as determined from the GIRSE analysis.

The relatively large uncertainties of the deformation potentials (Table III) stem mostly from the regression procedure itself. ${ }^{42}$ The regression does not account for the errors of the experimental strains and therefore may limit the accuracy with which the deformation potentials are determined [see Eq. (16) and Fig. 6]. The large uncertainties in the stiffness constants and the narrow frequency range could also contribute to large standard deviations of the deformation potentials. We note, however, that experimentally obtained phonon deformation potentials of GaN and AlN typically suffer from uncertainties as large as $20 \%-23 \% .^{27,43}$

We also have determined the $E_{1}(\mathrm{LO})$ phonon deformation potentials $a$ and $b$ by performing a regression analysis of the following equation:

$$
\begin{aligned}
\Delta \omega_{E_{1}(L O)}= & a_{E_{1}(L O)}\left(1-\frac{C_{11}}{C_{12}}\right) \epsilon_{x x}-\left(a_{E_{1}(L O)} \frac{C_{13}}{C_{12}}\right. \\
& \left.-b_{E_{1}(L O)}\right) \epsilon_{z z} \pm c_{E_{1}(L O)}\left|\epsilon_{x x}-\epsilon_{y y}\right|,
\end{aligned}
$$

obtained by substituting Eq. (3) in Eq. (6). The value of the $c_{E_{1}(L O)}$ from Table II was used as an input parameter. The obtained results are listed in Table III. The $a_{E_{1}(L O)}$ is determined with good accuracy. However, the larger uncertainties of the phonon frequencies and an insufficient number of data sensitive to the phonon anisotropy in the basal plane resulted in this case in a considerably larger error of the second deformation potential, $b_{E_{1}(L O)}$.

It is seen from Table III that there is very good agreement between the strain-free frequency values obtained by GIRSE and Raman scattering, ${ }^{26,44}$ whereas the theoretical values slightly deviate. We note that we have recently determined by Raman experiments strain-free frequencies of $530.7 \pm 0.1 \mathrm{~cm}^{-1}$ for the $A_{1}(\mathrm{TO})$ and $740.3 \pm 0.15 \mathrm{~cm}^{-1}$ for the $E_{1}(\mathrm{LO})$ phonons in a high-quality bulk GaN, which is in very good agreement with the present GIRSE results.

The $a_{A_{1}(T O)}$ obtained by GIRSE in the present work agrees well with the values previously determined by Raman scattering and theory. ${ }^{25,26}$ On the other hand, our $b_{A_{1}(T O)}$ is significantly larger than the theoretical estimation, ${ }^{25}$ confirming the experimental results of Davydov et al. ${ }^{26}$ There is very good agreement between the $a_{E_{1}(L O)}$ obtained by GIRSE in the present work and the theoretical estimation. Similarly to the $A_{1}(\mathrm{TO})$ mode, we found the second deformation potential of the $E_{1}(\mathrm{LO})$ to be significantly larger than the theoretically estimated $b_{A_{1}(T O)}$ (Table III). However, due to the large error, it is difficult to draw firm conclusions in this case. The reasons for the observed difference between the theoretical estimations and experimental determinations of the phonon deformation potentials are currently not well understood, but we note that a similar discrepancy between theory and experiment was found to be a general tendency for other phonon modes of GaN and AlN as well. ${ }^{25-27,43}$ In this respect it is important to emphasize that in our determination of the $A_{1}$ (TO) and $E_{1}(\mathrm{LO})$ deformation potentials only the experimental results about the strains and the phonon frequencies 

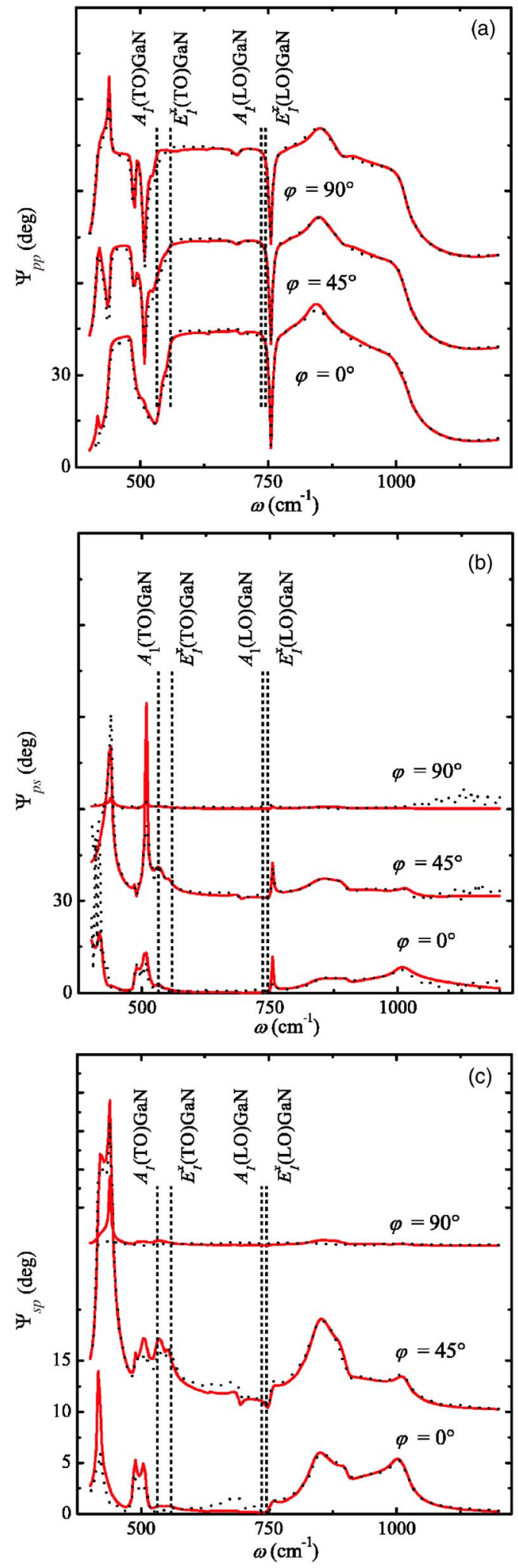

FIG. 5. (Color online) Experimental (dots) and calculated (lines) GIRSE spectra of one representative $a$-plane GaN on $r$-plane sapphire for different angles $\varphi$ between the plane of incidence and the GaN [0001] direction: (a) $\Psi_{p p}$, (b) $\Psi_{p s}$, and (c) $\Psi_{s p}$. The frequencies of the GaN phonon modes of $A_{1}$ and $E_{1}$ symmetry are indicated. For the sake of clarity the phonon frequencies are given only for polarization along the $[11 \overline{2} 0]$ direction.

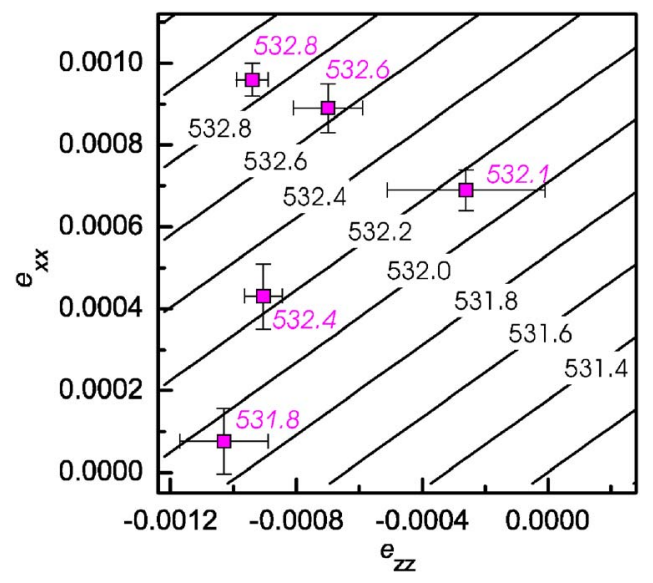

FIG. 6. (Color online) Contour plot of $A_{1}(\mathrm{TO})$ frequency versus the strain components $\epsilon_{x x}$ and $\epsilon_{z z}$ according to Eq. (16) and using our results on the phonon deformation potentials $\left(a=-664 \mathrm{~cm}^{-1}\right.$ and $\left.b=-1182 \mathrm{~cm}^{-1}\right)$ and strain-free frequency $\left(\omega_{0}=531.2 \mathrm{~cm}^{-1}\right)$. The squares show the calculated frequencies using our measured $\epsilon_{x x}$ and $\epsilon_{z z}$, and the respective labels give the frequency values as determined by GIRSE analysis.

were used without the need of additional hydrostatic experiments (usually performed on a different set of samples) or calibration of strain by other optical experiments. The latter are often used in the experimental determination of phonon deformation potentials of group-III nitrides and are considered as possible sources of errors. ${ }^{25}$ The uncertainties in the published stiffness constants of GaN, another source of possible errors in experimentally determined phonon deformation potentials, cannot account alone for the observed difference between theory and experiment. On the other hand, the choice of strain-free lattice parameters may significantly affect the experimental phonon deformation potentials and therefore most of the experimentally determined phonon deformation potentials of group-III nitrides may be biased, suffering from the lack of unification of strain-free lattice parameters of $\mathrm{GaN}(\mathrm{AlN}, \mathrm{InN})$.

\section{CONCLUSIONS}

The effect of anisotropic strain on GaN phonons was studied for $c$-plane $\mathrm{GaN}$ on $a$-plane sapphire using GIRSE complemented by Raman scattering spectroscopy. We have demonstrated the capability of the GIRSE to detect spectrally narrow dichroism, thereby allowing us to precisely locate the phonon mode resonances for different polarizations. As a result, we were able to identify splittings of the $\mathrm{GaN} E_{1}(\mathrm{TO})$ and $E_{1}(\mathrm{LO})$ phonon modes under anisotropic strain. We also demonstrate splitting of the GaN $E_{2}$ phonons measured by Raman spectroscopy. The observed splittings of the GaN phonons present experimental evidence of the theoretical predictions for wurtzite-structure group-III nitrides with anisotropic distortion of the basal plane. A correlation of phonon splittings with anisotropic strain components, which have been independently assessed by high-resolution $\mathrm{x}$-ray diffraction, allows us to estimate the $c$ phonon deformation potentials of the $\mathrm{GaN} E_{1}(\mathrm{TO})$ and $E_{1}(\mathrm{LO})$ phonons to be 
TABLE III. $A_{1}$ (TO) and $E_{1}(\mathrm{LO})$ strain-free frequency $\omega^{0}\left(\mathrm{in}^{-1}\right)$ and deformation potentials $a$ and $b$ (in $\mathrm{cm}^{-1}$ ) and the respective standard deviations.

\begin{tabular}{lcccccc}
\hline \hline & \multicolumn{3}{c}{$A_{1}(\mathrm{TO})$} & \multicolumn{3}{c}{$E_{1}(\mathrm{LO})$} \\
\cline { 2 - 6 } & $\omega_{A_{1}(\mathrm{TO})}^{0}$ & $a_{A_{1}(\mathrm{TO})}$ & $b_{A_{1}(\mathrm{TO})}$ & $\omega_{E_{1}(\mathrm{LO})}^{0}$ & $a_{E_{1}(\mathrm{LO})}$ & $b_{E_{1}(\mathrm{LO})}$ \\
\hline GIRSE, this work & $531.2 \pm 0.1$ & $-664 \pm 129$ & $-1182 \pm 282$ & $739.9 \pm 0.1$ & $-789 \pm 80$ & $-1169 \pm 532$ \\
Raman Expt. & $531.8^{\mathrm{a}}$ & $-630^{\mathrm{a}}$ & $-1290^{\mathrm{a}}$ & $742^{\mathrm{b}}$ & & \\
Theory & $540^{\mathrm{c}}$ & $-640^{\mathrm{c}}$ & $-695^{\mathrm{c}}$ & $757^{\mathrm{c}}$ & $-775^{\mathrm{c}}$ & $-703^{\mathrm{c}}$ \\
\hline \hline
\end{tabular}

${ }^{\mathrm{a}}$ Reference 26.

${ }^{\mathrm{b}}$ Reference 44.

${ }^{\mathrm{c}}$ Reference 25 .

$379 \pm 43 \mathrm{~cm}^{-1}$ and $682 \pm 49 \mathrm{~cm}^{-1}$, respectively, and an upper limit of $379 \pm 107 \mathrm{~cm}^{-1}$ for the $c_{E_{2}}$.

We have also studied the vibrational properties of $a$-plane GaN layers with different thicknesses in correlation with the anisotropic strain in the films, which allows us to determine by GIRSE the GaN $A_{1}$ (TO) and $E_{1}(\mathrm{LO})$ deformation potentials. The $a_{A_{1}(T O)}=-664 \pm 129 \mathrm{~cm}^{-1}$ and $b_{A_{1}(T O)}$ $=-1182 \pm 282 \mathrm{~cm}^{-1}$ are found to be in very good agreement with previous results from Raman scattering spectroscopy. The $E_{1}(\mathrm{LO})$ phonon deformation potentials have been experimentally determined to be $a_{E_{1}(L O)}=-789 \pm 80 \mathrm{~cm}^{-1}$ and $b_{E_{1}(L O)}=-1169 \pm 532 \mathrm{~cm}^{-1}$. Strain-free frequencies of $531.2 \pm 0.1 \mathrm{~cm}^{-1}$ and $739.9 \pm 0.1 \mathrm{~cm}^{-1}$ were determined for the $A_{1}(\mathrm{TO})$ and $E_{1}(\mathrm{LO})$ phonons, respectively. Good agreement of our results for $a_{A_{1}(T O)}$ and $a_{E_{1}(L O)}$ with recently reported theoretical estimations is found, while the $b_{A_{1}(T O)}$ and $b_{E_{1}(L O)}$ are significantly larger than the theoretical phonon deformation potentials. Thus, we confirm the recently reported large difference in $b_{A_{1}(T O)}$ as determined by theory and Raman scattering, respectively.

\section{ACKNOWLEDGMENTS}

V.D. would like to acknowledge support from the Swedish Research Council (VR) under Contract No. 2005-5054. M.S. acknowledges DFG research Grant No. SCHUH 1338/ 3-1, support by the J. A. Woollam Foundation, and starting funds from UNL.

\section{APPENDIX}

The Kramers Kronig relations ${ }^{45}$ connect the frequencydependent real and imaginary parts of the dielectric function $\varepsilon(\omega)=\varepsilon_{1}(\omega)+i \varepsilon_{2}(\omega)$,

$$
\varepsilon_{1}(\omega)-1=\frac{2}{\pi} P \int_{0}^{\infty} \frac{\omega^{\prime} \varepsilon_{2}\left(\omega^{\prime}\right)}{\omega^{\prime 2}-\omega^{2}} d \omega^{\prime},
$$

where $P$ indicates the main-value integral. Use of the geometrical series allows rearrangement of the denominator into the form

$$
\frac{1}{y}\left(1-\frac{x}{y}\right)^{-1}=\frac{1}{y} \sum_{\nu=0}^{\infty}\left(\frac{x}{y}\right)^{\nu}
$$

For $\omega \rightarrow \infty$ and with $\varepsilon_{2}(\omega)$ being a continuously differentiable function that vanishes faster than $\omega^{-1}$, the superconvergence theorem suggested by Altarelli et al. ${ }^{46}$ can be easily recovered:

$$
\varepsilon_{1}(\omega)-1 \approx \frac{2}{\pi \omega^{2}} P \int_{0}^{\infty} \omega^{\prime} \varepsilon_{2}\left(\omega^{\prime}\right) d \omega^{\prime}, \quad \omega \rightarrow \infty .
$$

A valuable consequence for ellipsometry measurements of small perturbations in physical material properties can be obtained here. Let such perturbations result in variations $\delta \varepsilon_{1}(\omega)$ and $\delta \varepsilon_{2}(\omega)$. Then $\delta \varepsilon_{2}$ may represent a small and spectrally narrow perturbation of the absorption coefficient-for example, due to a small phonon mode shift with absorption line centered at $\omega_{0}$. With $\varepsilon \rightarrow \varepsilon+\delta \varepsilon_{1}+i \delta \varepsilon_{2}$ being the dielectric function of the perturbed system the effect of $\delta \varepsilon_{1}(\omega)$ according to Eq. (20) is that of the superposition of a long tail onto $\varepsilon_{1}(\omega)$ with the same slope as a Lorentzian oscillator

$$
\delta \varepsilon_{1}(\omega) \approx \frac{\text { const }}{\omega^{2}}, \quad \omega \gg \omega_{0}
$$

Although strictly valid for frequencies far away from any resonance frequency region only, however, the main conclusion is constituted by the fact that small phonon mode frequency shifts can be traced by measurement of the index of refraction within spectral regions far away from the actual resonance. Hence, the resolution capability of the ellipsometer instrument must not necessarily be better than the actual phonon resonance line shift since its influence on the real part of the dielectric function is detectable over a much larger spectral range. If perturbations cause different shifts for different spatial directions, the effects are a spectrally narrow dichroism and wide birefringence, which may be best detected and evaluated by the generalized ellipsometry approach. 
*Corresponding author. FAX: +46 13 142337. Electronic address: vanya@ifm.liu.se

†Now with Osram Opto Semiconductors, Germany.

Now at Ulm University, Germany.

${ }^{1}$ M. D. Craven, S. H. Lim, F. Wu, J. S. Speck, and S. P. DenBaars, Appl. Phys. Lett. 81, 469 (2002).

${ }^{2}$ M. D. Craven, F. Wu, A. Chakraborty, B. Imer, U. K. Mishra, S. P. DenBaars, and J. S. Speck, Appl. Phys. Lett. 84, 1281 (2004).

${ }^{3}$ M. McLaurin, T. E. Mates, and J. S. Speck, Appl. Phys. Lett. 86, 262104 (2005).

${ }^{4}$ P. Waltereit, O. Brandt, A. Trampert, H. T. Grahn, J. Menniger, M. Ramsteiner, M. Reiche, and K. H. Ploog, Nature (London) 406, 865 (2000).

${ }^{5}$ N. Akopian, G. Bahir, D. Gershoni, M. D. Craven, J. S. Speck, and S. P. DenBaars, Appl. Phys. Lett. 86, 202104 (2005).

${ }^{6}$ D. Doppalapudi, E. Ilioulos, S. N. Basu, and T. D. Moustakas, J. Appl. Phys. 85, 3582 (1999).

${ }^{7}$ S. Nakamura, M. Senoh, S. Nagahama, N. Iwasa, T. Yamada, T. Matsushita, H. Kiyoki, and Y. Sigimoto, Jpn. J. Appl. Phys., Part 2 35, L217 (1996).

${ }^{8}$ A. Alemu, B. Gil, M. Julier, and S. Nakamura, Phys. Rev. B 57, 3761 (1998).

${ }^{9}$ P. P. Paskov, V. Darakchieva, T. Paskova, P. O. Holtz, and B. Monemar, Phys. Status Solidi B 234, 892 (2002).

${ }^{10}$ P. P. Paskov, R. Schifano, B. Monemar, T. Paskova, S. Figge, and D. Hommel, J. Appl. Phys. 98, 093519 (2005).

${ }^{11}$ S. Ghosh, P. Misra, H. T. Grahn, B. Ilmer, S. Nakamura, S. P. DenBaars, and J. S. Speck, J. Appl. Phys. 98, 026105 (2005).

${ }^{12}$ S. Ghosh, P. Waltereit, O. Brandt, H. T. Grahn, and K. H. Ploog, Phys. Rev. B 65, 075202 (2002).

${ }^{13}$ B. Rau, P. Waltereit, O. Brandt, M. Ramsteiner, K. H. Ploog, J. Puls, and F. Henneberger, Appl. Phys. Lett. 77, 3343 (2000).

${ }^{14}$ S. Ghosh, P. Waltereit, O. Brandt, H. T. Grahn, and K. H. Ploog, Appl. Phys. Lett. 80, 413 (2002).

${ }^{15}$ V. Darakchieva, P. P. Paskov, T. Paskova, E. Valcheva, B. Monemar, and M. Heuken, Appl. Phys. Lett. 82, 703 (2003).

${ }^{16}$ H. Wang, C. Chen, Z. Gong, J. Zhang, M. Gaewski, M. Su, J. Yang, and M. Asif Khan, Appl. Phys. Lett. 84, 499 (2004).

${ }^{17}$ T. Paskova, V. Darakchieva, P. P. Paskov, J. Birch, E. Valcheva, P. O. A. Persson, B. Arnaudov, S. Tungasmita, and B. Monemar, J. Cryst. Growth 281, 55 (2005).

${ }^{18}$ Y. Takagaki, C. Hucho, E. Wiebicke, Y. J. Sun, O. Brandt, M. Ramsteiner, and K. H. Ploog, Phys. Rev. B 69, 115317 (2004).

${ }^{19}$ O. Brandt, Y. J. Sun, L. Däweritz, and K. H. Ploog, Phys. Rev. B 69, 165326 (2004).

${ }^{20}$ D. N. Zakharov, Z. Liliental-Weber, B. Wagner, Z. J. Reitmeier, E. A. Preble, and R. F. Davis, Phys. Rev. B 71, 235334 (2005).

${ }^{21}$ O. Brandt, Y. J. Sun, L. Daweritz, and K. H. Ploog, Phys. Rev. B 69, 165326 (2004).

${ }^{22}$ M. E. Twigg, R. L. Henry, A. E. Wickenden, D. D. Koleske, and J. C. Culbertson, Appl. Phys. Lett. 75, 686 (1999).
${ }^{23}$ T. Paskova, E. M. Goldys, and B. Monemar, J. Cryst. Growth 203, 1 (2000).

${ }^{24}$ R. J. Briggs and A. K. Ramdas, Phys. Rev. B 13, 5518 (1976).

${ }^{25}$ J.-M. Wagner and F. Bechstedt, Phys. Rev. B 66, 115202 (2002).

${ }^{26}$ V. Yu. Davydov, N. S. Averkiev, I. N. Goncharuk, D. K. Nelson, I. P. Nikitina, A. S. Polkovnikov, A. N. Smirnov, M. A. Jacobson, and O. K. Semchinova, J. Appl. Phys. 82, 5097 (1997).

${ }^{27}$ F. Demangeot, J. Frandon, P. Baules, F. Natali, F. Semond, and J. Massies, Phys. Rev. B 69, 155215 (2004).

${ }^{28}$ J. Off, Ph. D. thesis (Shaker Verlag, Aachen, 2001).

${ }^{29}$ B. A. Haskell, F. Wu, S. Matsuda, M. D. Craven, P. T. Fini, S. P. DenBaars, J. S. Speck, and S. Nakamura, Appl. Phys. Lett. 83, 1554 (2003).

${ }^{30}$ V. Darakchieva, J. Birch, M. Schubert, T. Paskova, S. Tungasmita, G. Wagner, A. Kasic, and B. Monemar, Phys. Rev. B 70, 045411 (2004).

${ }^{31}$ H. Angerer, D. Brunner, F. Freudenberg, O. Ambacher, M. Stutzmann, R. Höpler, T. Metzger, E. Born, G. Dollinger, A. Bergmaier, S. Karsch, and H.-J. Körner, Appl. Phys. Lett. 71, 1504 (1997).

${ }^{32}$ A. E. Romanov, T. J. Baker, S. Nakamura, and J. S. Speck, J. Appl. Phys. 100, 023522 (2006).

${ }^{33}$ M. Schubert, Infrared Ellipsometry on Semiconductor Layer Structures: Phonons, plasmons and polaritons (Springer, Heidelberg, 2004), Vol. 209.

${ }^{34}$ M. Schubert, Phys. Rev. B 53, 4265 (1996).

${ }^{35}$ M. Schubert, B. Rheinlander, J. A. Woollam, B. Johs, and C. M. Herzinger, J. Opt. Soc. Am. A 13, 875 (1996).

${ }^{36}$ M. Schubert, Thin Solid Films 313-314, 323 (1998).

${ }^{37}$ M. Schubert, T. E. Tiwald, and C. M. Herzinger, Phys. Rev. B 61, 8187 (2000).

${ }^{38}$ A. Kasic, M. Schubert, S. Einfeldt, D. Hommel, and T. E. Tiwald, Phys. Rev. B 62, 7365 (2000).

${ }^{39}$ M. Leszczynski, T. Suski, H. Teisseyre, P. Perlin, I. Grzegory, J. Jun, and S. Porowski, J. Appl. Phys. 76, 4909 (1994).

${ }^{40}$ M. Yamaguchi, T. Yagi, T. Azuhata, T. Sota, K. Suzuki, S. Chichibu, and S. Nakamura, J. Phys.: Condens. Matter 9, 241 (1997).

${ }^{41}$ A. Kasic, D. Gogova, H. Larsson, C. Hemmingsson, I. Ivanov, B. Monemar, C. Bundesmann, and M. Schubert, Phys. Status Solidi A A201 2773 (2004).

${ }^{42}$ M. G. Kendall and A. Stuart, The Advanced Theory of Statistics (Charles Griffin, London, 1961), Vol. 2.

${ }^{43}$ J. Gleize, M. A. Renucci, J. Frandon, E. Ballet-Amalric, and B. Daudin, J. Appl. Phys. 93, 2065 (2003).

${ }^{44}$ A. R. Goñi, H. Siegle, K. Syassen, C. Thomsen, and J. M. Wagner, Phys. Rev. B 64, 035205 (2001).

${ }^{45}$ M. Dressel, G. Güner, Electrodynamics of Solids (Cambridge University Press, Cambridge, England, 2002).

${ }^{46}$ M. Altarelli, D. L. Dexter, H. M. Nussenzweig, and D. Y. Smith, Phys. Rev. B 6, 4502 (1972). 